\title{
The roles of shear and cross-correlations on the fluctuation levels in simple stochastic models
}

\author{
John A. Krommes \\ Princeton University, P. O. Box 451, Princeton, NJ 08543-0451 USA
}

(December 8, 1999)

\begin{abstract}
Highly simplified models of random flows interacting with background microturbulence are analyzed. In the limit of very rapid velocity fluctuations, it is shown rigorously that the fluctuation level of a passively advected scalar is not controlled by the rms shear. In a model with random velocities dependent only on time, the level of cross-correlations between the flows and the background turbulence regulates the saturation level. This effect is illustrated by considering a simple stochasticoscillator model, both exactly and with analysis and numerical solutions of the direct-interaction approximation. Implications for the understanding of self-consistent turbulence are discussed briefly.
\end{abstract}

PACS: 52.35.Ra

\section{INTRODUCTION}

It is widely believed that random zonal flows play an important role in the regulation of the saturation and transport levels of plasma microturbulence. In a Cartesian geometry with wave number $\boldsymbol{k} \equiv\left(k_{x}, k_{y}, k_{z}\right)$, zonal flows are defined to be fluctuations with $k_{y}=k_{z}=0$. An electrostatic potential fluctuation $\delta \varphi(x, t)$ creates an $\boldsymbol{E} \times \boldsymbol{B}$ flow in the $y$ direction: $\delta V_{E, y}(x, t)=\partial_{x} \varphi$. Such flows are sheared, $\partial_{x} \delta V_{E, y} \neq 0$. For time-independent shear, it is well known that the shearing effect can influence correlation times and/or lengths and the levels of fluctuations and/or transport. ${ }^{1}$ Recently Hahm et al. ${ }^{2}$ studied the effect on correlation lengths of zonal flows oscillating in time; they found that shearing effects would be reduced by rapidly oscillating zonal flows. They argued that the total rms shearing rate

$$
\omega_{s} \doteq\left(\sum_{\boldsymbol{k}} k^{2}\left\langle\left|\delta \boldsymbol{V}_{\boldsymbol{k}}\right|^{2}\right\rangle\right)^{1 / 2},
$$

where $\delta \boldsymbol{V}_{\boldsymbol{k}}$ is the Fourier amplitude of the velocity field and $\doteq$ means definition, should not be used in estimates of fluctuation level and proposed a formula for an effective rate $\omega_{\text {eff }}$ that, although scaling with $\omega_{s}$, was reduced from $\omega_{s}$ by rapid time variations. Analysis of various computer simulations appeared to support their conclusion: although $\omega_{s}$ was far greater than the linear growth rate $\gamma$, the turbulence appeared to saturate at a level for which $\omega_{\text {eff }}$ was of the order of $\gamma$.

Hahm et al. studied the effects of coherent (statistically sharp) zonal flows on a passively advected scalar field $\psi$. Recognizing that coherency was a poor assumption, they called for a statistical analysis. In the present paper I describe some introductory aspects of such an analysis. The work should not be thought of as focusing primarily on the details of zonal flows, for which a number of important details are modeled inadequately, but rather as discussing some interesting properties of random flows and associated stochastic models.
One important goal of a statistical formalism is calculation of the turbulent flux $\Gamma$. The value of $\Gamma$ is determined by a variety of effects, including the overall fluctuation level (two-point correlations that are diagonal in the field index); phase relations (off-diagonal correlations); and spectral distributions, determined by nonlinear mode-coupling processes. Although in a fully selfconsistent theory all of these effects are determined simultaneously, it is instructive to study them independently. The present paper concentrates on some properties of fluctuation levels; it does not address the calculation of $\Gamma$.

The method I shall use involves analyses of highly simplified stochastic models. In a stochastic dynamical model, some details of the true nonlinear dynamics are modified to include additional, externally specified stochastic attributes that change the total probability density functional of the turbulence (particularly in the higher moments), but hopefully retain some reasonable information about low-order (e.g., two-point) statistics. The use of stochastic models is well accepted in general turbulence theory ${ }^{3}$ for its pedagogical value and for providing rigorous benchmarks against which more detailed calculations must be compared. (A recent example of the use of a particular kind of stochastic model can be found in Ref. 4.) However, it must be emphasized that the models studied here are significantly lacking in physical detail, so the present calculations are highly incomplete and unsatisfying. They must be viewed as a way of illustrating some basic conceptual points with a minimum of complication. For applications, much more realistic and involved calculations must be done; such work will be presented elsewhere.

An outline of the paper is as follows. In Sec. II I introduce a general model of passively advected turbulence. Then, motivated by the discussion of rapidly varying, coherent zonal flows in Ref. 2, I consider the so-called rapidchange model of random passive advection, in which the stochastic velocity field varies very rapidly in time. In that limit, which was discussed thoroughly in Ref. 5, it follows immediately and rigorously that the rms shear 
does not control the saturation level at all; that level is nonzero even in the limit of infinitely rapid velocity variations. I then consider to what extent nontrivial results about fluctuation levels can follow from stochastic models that involve the addition of spatially independent (hence unsheared) velocity fields to a randomly forced turbulent background. This is discussed in Sec. III, which contains the bulk of the paper. When the velocities are uncorrelated with the random forcing, that basic question is quickly answered in the negative (Sec. III A); such velocities do not change the fluctuation level. However, I show that a reduction in the saturation level can be found by imposing statistical correlations between the explicit flows and the background turbulence. To illustrate this, I analyze a simple model, both exactly (Sec. III B) and with the direct-interaction approximation (Sec. III C). Sec. IV contains a brief discussion.

\section{PASSIVE ADVECTION, RAPID CHANGE, AND RMS SHEAR}

Following current practice in the plasma-physics literature on shear-related effects, I shall consider the passive advection of a scalar $\psi$ such as temperature. To keep the discussion as general as possible, I do not attempt to model detailed linear physics, but rather encapsulate it in a random external forcing $\delta f^{(\text {ext })}$ and classical dissipation $\eta_{\mathrm{cl}}=-D_{\mathrm{cl}} \nabla^{2}$. One may think of the gradients as acting in the direction perpendicular to a strong magnetic field. Since parallel dynamics will not be considered explicitly, the effective spatial dimensionality is $d=2$. For simplicity, I assume homogeneous statistics, so statistical observables depend only on spatial differences. Furthermore, in order to clearly restrict the focus to fluctuation levels, I shall assume isotropic statistics (which requires, in particular, the absence of a mean field with gradient in, say, the $x$ direction). The mean flux $\Gamma \doteq\left\langle\delta V_{E, x} \delta \psi\right\rangle$ therefore vanishes, although the fluctuation level is nonzero (and fluctuations are still damped, at a rate $\eta$ that includes both turbulent and classical contributions). Passively advected fluctuations then obey

$$
\partial_{t} \delta \psi(\boldsymbol{x}, t)+\delta \boldsymbol{V}(\boldsymbol{x}, t) \cdot \boldsymbol{\nabla} \delta \psi-D_{\mathrm{cl}} \nabla^{2} \delta \psi=\delta f^{(\mathrm{ext})}(\boldsymbol{x}, t),
$$

where $\delta \boldsymbol{V}$ is a statistically specified random velocity (with zero mean) that shall in the present section be taken to be statistically independent of $\delta f^{(\mathrm{ext})}$. (This conventional assumption will be relaxed in Sec. III.) $\delta \boldsymbol{V}$ may have an arbitrary Eulerian Fourier wave-number spectrum $\delta \boldsymbol{V}_{\boldsymbol{k}}$, possibly including zonal flows. The assumption of random flows, with a given autocorrelation time $\tau_{\mathrm{ac}}$, generalizes the model studied in Ref. 2 of coherent flows oscillating with a frequency $\omega_{f} \sim \tau_{\mathrm{ac}}^{-1}$.

In general, the two-point statistics of the forced, dissipative steady states achieved by Eq. (2) can be adequately treated by the direct-interaction approximation
(DIA) or related Markovian approximations. (For discussion and original references on the DIA and related closures, see Ref. 6.) However, it is very instructive to consider the so-called rapid-change model ${ }^{7}$ in which both the velocity field and the random forcing vary infinitely rapidly in time. One takes

$$
\begin{gathered}
\langle\delta \boldsymbol{V}(\boldsymbol{x}+\boldsymbol{\rho}, t+\tau) \delta \boldsymbol{V}(\boldsymbol{x}, t)\rangle=2\langle\delta \boldsymbol{V} \delta \boldsymbol{V}\rangle(\rho) \tau_{\mathrm{ac}} \delta(\tau), \\
\left\langle\delta f^{(\mathrm{ext})}(\boldsymbol{x}+\boldsymbol{\rho}, t+\tau) \delta f^{(\mathrm{ext})}(\boldsymbol{x}, t)\right\rangle=F^{(\mathrm{ext})}(\rho) \delta(\tau) .
\end{gathered}
$$

In Eq. (3a), $\tau_{\text {ac }}$ is assumed to be much smaller than an eddy turnover time for $\delta \psi$ and formally goes to zero; however, in that limit $^{8}$ the velocities must be rescaled such that the turbulent diffusion coefficient

$$
D_{\text {turb }}=\tau_{\text {ac }} \sum_{\boldsymbol{k}}\left\langle\left|\delta \boldsymbol{V}_{\boldsymbol{k}}\right|^{2}\right\rangle
$$

remains finite and nonzero, as does $F^{(\text {ext })}(0) \doteq \sigma$. [It will be seen from Eq. (5) that $\sigma$ is the externally injected production of scalar variance.] Then the equation for the two-space-point, equal-time correlation function $C(\boldsymbol{\rho}, t) \doteq\langle\delta \psi(\boldsymbol{x}+\boldsymbol{\rho}, t) \delta \psi(\boldsymbol{x}, t)\rangle$ can be shown $^{7}$ to rigorously close. For homogeneous, isotropic statistics one finds

$$
\partial_{t} C(\rho, t)-\frac{2}{\rho^{d-1}} \frac{\partial}{\partial \rho} \rho^{d-1} D_{-}(\rho) \frac{\partial C}{\partial \rho}=F^{(\mathrm{ext})}(\rho) .
$$

Here $D_{-}(\rho) \doteq \widehat{D}_{-}(\rho)+D_{\text {cl }}$, where

$$
\widehat{D}_{-}(\rho) \doteq \tau_{\mathrm{ac}} \sum_{\boldsymbol{k}}\left(1-e^{i \boldsymbol{k} \cdot \boldsymbol{\rho}}\right)\left\langle\left|\delta \boldsymbol{V}_{\boldsymbol{k}}\right|^{2}\right\rangle .
$$

The content of Eq. (5) was discussed at length in Ref. 5, where a number of misconceptions were identified. In particular, although it has been frequently suggested in the literature that the steady states of Eq. (5) are controlled by the so-called clump lifetime ${ }^{9} \tau_{\mathrm{cl}}(\rho)$, a property of the very small scales, it was shown in Ref. 5 that this is almost never the case. An equivalent statement is that the Taylor velocity microscale $\lambda_{\mathrm{T}} \doteq k_{T}^{-1}$, a measure [see Eq. (9)] of the rms velocity shear (hence dominated by the small scales), does not determine the steady-state fluctuation level. Instead, that level is determined by properties of the large, energy-containing scales. This can be readily seen from the steady-state solution of Eq. (5), which specialized to the intensity $I \doteq C(0, t=\infty)$ is

$$
\begin{aligned}
I & =\lim _{\rho \rightarrow 0} C(\rho), \\
C(\rho) & =\frac{1}{2} \int_{\rho}^{\infty} \frac{d \bar{\rho}}{\bar{\rho}^{d-1} D_{-}(\bar{\rho})} \int_{0}^{\bar{\rho}} d \bar{\rho}^{\prime} \bar{\rho}^{\prime d-1} F^{(\mathrm{ext})}\left(\bar{\rho}^{\prime}\right) .
\end{aligned}
$$

Because $\lim _{\rho \rightarrow \infty} D_{-}(\rho)=D \doteq D_{\text {turb }}+D_{\text {cl }}$, the $\bar{\rho}$ integral would be divergent at large scales were it not for the cutoff afforded by the $\bar{\rho}^{\prime}$ integral over $F^{\text {(ext) }}$, which is assumed to occur at a macroscopic scale $L_{\mathrm{c}}$. As discussed 
in Ref. 5 and can easily be seen on dimensional grounds, the final result is

$$
I=\mathcal{O}\left(\sigma \tau_{D}\right),
$$

where $\tau_{D} \doteq L_{\mathrm{c}}^{2} / D$ is the diffusion time based on $L_{\mathrm{c}}$. This formula does not depend on

$$
-\left.\frac{\partial^{2} D_{-}(\rho)}{\partial \boldsymbol{\rho} \cdot \partial \boldsymbol{\rho}}\right|_{\rho=0}=\tau_{\mathrm{ac}} \sum_{\boldsymbol{k}} k^{2}\left\langle\left|\delta \boldsymbol{V}_{\boldsymbol{k}}\right|^{2}\right\rangle=\tau_{\mathrm{ac}} \omega_{s}^{2} \equiv k_{T}^{2} D
$$

- that is, it does not depend on the rms flow shear or on the Taylor microscale. It does, of course, depend on the Fourier spectrum of the large, energy-containing scales through its dependence on $D_{\text {turb }}$.

By considering the dynamics of small-scale eddies and by analyzing a variety of computer simulations, Hahm et al. also concluded that saturation level did not appear to be controlled by the total flow shear. Within their framework of coherently oscillating flows of frequency $\omega_{f}$, they used a theory of small-scale eddy distortions due to shear to derive a formula for an effective modal shearing rate $\omega_{\text {eff }}(\boldsymbol{k})$ that was reduced from the raw rms shearing rate $\omega_{s}$ when $\zeta \doteq\left(\omega_{f} / \omega_{t}\right)^{2} \gg 1\left(\omega_{t}\right.$ is the turbulent decorrelation time). Their result had the structural form

$$
\omega_{\mathrm{eff}} / \omega_{s}=g(\zeta), \quad g(0)=1, g(\infty)=0,
$$

where $g(\zeta)$ is given by Eq. (13) of Ref. 2. Their data appeared to be consistent with the balance $\sqrt{N} \omega_{\text {eff }}(\boldsymbol{k}) \sim$ $\gamma_{\boldsymbol{k}}, N$ being the total number of zonal flow modes and entering because of an assumption of incoherent superposition of modes. It is difficult to compare the predictions of Ref. 2 with those of the present section because of (i) the differing assumptions of coherent vs random advection; and (ii) the approximate nature of the eddy-distortion model used in Ref. 2 [recall that within the confines of the rapid-change model, Eqs. (5)-(8) are exact]. However, it should be noted that the fluctuation level in the rapid-change model does not involve $\omega_{s}$ at all, whereas predictions of $\omega_{\text {eff }}$ from Eq. (10) scale with $\omega_{s}$. Furthermore, $\omega_{\text {eff }} \rightarrow 0$ as $\omega_{f} \rightarrow \infty$, so $\omega_{\text {eff }}$ cannot be relevant to the fluctuation level in that limit. To estimate the rapid-change saturation level, note that if one approximates $\sigma \sim 2 \gamma I$, where $\gamma$ is a typical growth rate, then Eq. (8) reads

$$
\tau_{D}^{-1} \sim \gamma, \text { or } D \sim \gamma / \bar{k}^{2},
$$

where $\bar{k}=L_{\mathrm{c}}^{-1}$. This is a familiar estimate, although it is usually written with $\bar{k}$ replaced by an unspecified $k$. The present derivation makes it clear that $\bar{k}$ is not arbitrary, but is the correlation length of the turbulence. Note that Eq. (11b) makes a statement only about typical values, not detailed wave-number dependence. In principle, such dependence can be obtained from the Fourier transform of Eq. (7b). Unfortunately, insufficient data is presented in Ref. 2 for one to attempt to evaluate formula (7b) even approximately. Although that would be an instructive exercise, it must be emphasized that (i) the simulations (and physical experiments) are self-consistent, not passive; (ii) in reality, the velocity fields do not vary infinitely rapidly; (iii) as consequences of (i) and (ii), Eq. (5) does not rigorously hold and, even if it did, an accurate representation of the effective, self-consistent $F^{(\mathrm{ext})}(\rho)$ is not known. Therefore, without significant additional analytical and numerical work it is not possible to make an accurate calculation of the fluctuation spectrum, because the large-scale dynamics that control the saturation level involve mode couplings of comparable scales that do not yield to perturbation expansion. (This same remark would apply to transport levels, which are not treated in the present model.) Numerical solution of various self-consistent models in the DIA is feasible, but is not attempted here. But the general conclusion, that the rms shear does not control the fluctuation level, is robust. It transcends the rapid-change model,,$^{5}$ because the final prediction $(7 \mathrm{~b})$ for the spectrum involves only the diffusion coefficient (a macroscopic property of the turbulence), not the details of $\tau_{\mathrm{ac}}$ or $\delta \boldsymbol{V}_{\boldsymbol{k}}$ separately.

\section{THE EFFECTS OF CROSS-CORRELATIONS}

Although realistic studies of physical turbulence require considerable additional analysis of self-consistent nonlinearities, it is conceptually interesting to ask what is the minimal model that makes nontrivial predictions for fluctuation levels. In the present section I inquire about the properties of simple stochastic models of passive advection that involve velocity fields dependent only on time (thus possessing no shear whatsoever). That is, I will study variants of the equation

$$
\partial_{t} \delta \psi+\delta \boldsymbol{V}(t) \cdot \nabla \delta \psi+\eta \delta \psi=\delta f
$$

where $\eta$ represents turbulent and classical damping of the background turbulence and $\delta f$ represents the statistically consistent forcing of that background (including not only the "external" contribution discussed in the previous section but also an internal contribution from incoherent mode coupling). Unfortunately, one encounters the immediate difficulty that when $\delta \boldsymbol{V}$ and $\delta f$ are uncorrelated it is clear, on general grounds of random Galilean invariance, and can easily be shown (Sec. III A) that the contributions of space-independent velocity fields do not affect the fluctuation level at all. For example, in the rapid-change model such contributions disappear from Eq. (7b) because $\boldsymbol{k}=0$ Fourier components do not contribute to $D_{-}(\rho)$. [With respect to infinitely longwavelength fluctuations, any $\rho$ is relatively very small, and $D_{-}(\rho=0)=0$.] Another way of saying this is that Eq. (8) depends only on the $\boldsymbol{k} \neq 0$ velocity spectrum.

Evidently models such as Eq. (12) exhibit nontrivial fluctuation levels only if cross-correlations between $\delta \boldsymbol{V}$ 
and $\delta f$ are allowed. In Secs. III B and III C I shall explore the consequences of such a model. To set the stage, I first provide in the next section some background about Langevin models for turbulence.

\section{A. Langevin models including random zonal flows and background turbulence}

Let the background turbulence be described by the general class of Markovian statistical closures represented by the Langevin equation

$$
\partial_{t} \delta \psi(\boldsymbol{x}, t)+\eta(\boldsymbol{x}) \star \delta \psi(\boldsymbol{x}, t)=\delta f(\boldsymbol{x}, t),
$$

where $A(\boldsymbol{x}) \star B(\boldsymbol{x}) \doteq \int_{-\infty}^{\infty} d \overline{\boldsymbol{x}} A(\boldsymbol{x}-\overline{\boldsymbol{x}}) B(\overline{\boldsymbol{x}})$ and $\delta f(\boldsymbol{x}, t)$ is a random stirring, delta-correlated in time, that is statistically consistent with the turbulent (plus linear) damping $\eta$ :

$$
\begin{aligned}
\left\langle\delta f(\boldsymbol{x}, t) \delta f\left(\boldsymbol{x}^{\prime}, t^{\prime}\right)\right\rangle & \doteq F\left(\boldsymbol{x}, t ; \boldsymbol{x}^{\prime}, t^{\prime}\right) \\
& =F\left(\boldsymbol{x}-\boldsymbol{x}^{\prime}\right) \delta\left(t-t^{\prime}\right),
\end{aligned}
$$

where $F(\boldsymbol{\rho})$ is a certain function. In a particular statistical closure ${ }^{10,6}$ such as the eddy-damped quasi-normal Markovian (EDQNM) approximation, ${ }^{11}$ specific formulas for $\eta$ and $F$ are given (usually for their Fourier transforms with respect to $\left.\boldsymbol{\rho} \doteq \boldsymbol{x}-\boldsymbol{x}^{\prime}\right)$. The detailed expressions are not required here; the only (strong and unrealistic) assumption is that they are unchanged when zonal flows are added to the dynamics

The assumption that $\delta f(\boldsymbol{\rho}, \tau)\left(\tau \doteq t-t^{\prime}\right)$ is deltacorrelated is significant. Although it is rigorously true for the EDQNM, ${ }^{12}$ it does not hold for more sophisticated closures such as the realizable Markovian closure ${ }^{13}$ or the direct-interaction approximation. ${ }^{14}$ As will be seen, it is used here as a device to cleanly separate effects of crosscorrelations from more subtle effects involving the shapes of correlation functions that are not known accurately within the confines of the present theory.

\section{Fluctuation levels in Langevin models}

To find the fluctuation level of such background turbulence, one can form the spectral balance equation for the two-point correlation function

$$
C\left(\boldsymbol{x}, t ; \boldsymbol{x}^{\prime}, t^{\prime}\right) \doteq\left\langle\delta \psi(\boldsymbol{x}, t) \delta \psi\left(\boldsymbol{x}^{\prime}, t^{\prime}\right)\right\rangle \equiv C(t \mid \boldsymbol{\rho}, \tau)
$$

by multiplying Eq. (13) by $\delta \psi\left(\boldsymbol{x}^{\prime}, t^{\prime}\right) \equiv \delta \psi^{\prime}$ :

$$
\partial_{t} \delta \psi \delta \psi^{\prime}+\eta \star \delta \psi \delta \psi^{\prime}=\delta f \delta \psi^{\prime} .
$$

Upon ensemble-averaging Eq. (16) and assuming periodic boundary conditions (compatible with statistical homogeneity) and stationary forcing, one finds

$$
\partial_{t} C(t \mid \boldsymbol{\rho}, \tau)+\eta \star C=W(t \mid \boldsymbol{\rho}, \tau),
$$

where

$$
W(t \mid \boldsymbol{\rho}, \tau) \doteq\left\langle\delta f(\boldsymbol{x}, t) \delta \psi\left(\boldsymbol{x}^{\prime}, t^{\prime}\right)\right\rangle
$$

describes the statistical effect of the random forcing. The fluctuation level is $I=C(t \mid \mathbf{0}, 0)=\sum_{\boldsymbol{k}} C_{\boldsymbol{k}}(t)$, where $C_{\boldsymbol{k}}(t)$ is the Fourier transform of $C(t \mid \boldsymbol{\rho})$ and $C(t \mid \boldsymbol{\rho}) \equiv C(t \mid \boldsymbol{\rho}, \tau=0)$. The equation for the equaltime correlation function follows from Eq. (17) as

$$
\begin{aligned}
\partial_{t} C(t \mid \boldsymbol{\rho}) & +\left[\eta(\boldsymbol{x}) \star C(t \mid \boldsymbol{\rho})+\left(\boldsymbol{x} \leftrightarrow \boldsymbol{x}^{\prime}\right)\right] \\
& =2 W(t \mid \boldsymbol{\rho}, 0)
\end{aligned}
$$

To calculate $W$, one may introduce the Green's function (infinitesimal response function) $R$, which obeys

$$
\partial_{t} R\left(\boldsymbol{x}, t ; \boldsymbol{x}^{\prime}, t^{\prime}\right)+\eta \star R=\delta\left(\boldsymbol{x}-\boldsymbol{x}^{\prime}\right) \delta\left(t-t^{\prime}\right) .
$$

[ $R$ is statistically sharp because the left-hand sides of Eqs. (13) or (20) contain no random elements.] Then

$$
\delta \psi\left(\boldsymbol{x}^{\prime}, t^{\prime}\right)=\int_{-\infty}^{t^{\prime}} d \bar{t} \int_{-\infty}^{\infty} d \overline{\boldsymbol{x}} R\left(\boldsymbol{x}^{\prime}, t^{\prime} ; \overline{\boldsymbol{x}}, \bar{t}\right) \delta f(\overline{\boldsymbol{x}}, \bar{t})
$$

(a transient contribution from initial conditions is ignored) and

$$
W(t \mid \boldsymbol{\rho}, 0)=\int_{-\infty}^{t} d \bar{t} \int_{-\infty}^{\infty} d \bar{x}\left\langle\delta f(\boldsymbol{x}, t) R\left(\boldsymbol{x}^{\prime}, t ; \overline{\boldsymbol{x}}, \bar{t}\right) \delta f(\overline{\boldsymbol{x}}, \bar{t})\right\rangle .
$$

Since $R$ is statistically sharp, the ensemble average rigorously factors:

$$
\left\langle\delta f(\boldsymbol{x}, t) R\left(\boldsymbol{x}^{\prime}, t ; \overline{\boldsymbol{x}}, \bar{t}\right) \delta f(\overline{\boldsymbol{x}}, \bar{t})\right\rangle=F(\boldsymbol{x}, t ; \overline{\boldsymbol{x}}, \bar{t}) R\left(\boldsymbol{x}^{\prime}, t ; \overline{\boldsymbol{x}}, \bar{t}\right) .
$$

Upon recalling that $F$ is delta-correlated in time, one can perform the integral in Eq. (22) to find

$$
W(t \mid \boldsymbol{\rho}, 0)=\frac{1}{2} F(\boldsymbol{\rho}) .
$$

Here one noted that $R(\boldsymbol{x}, t+\epsilon ; \overline{\boldsymbol{x}}, t)=\delta(\boldsymbol{x}-\overline{\boldsymbol{x}})$ or, equivalently, that $R(\boldsymbol{x}, t ; \overline{\boldsymbol{x}}, t)=\frac{1}{2} \delta(\boldsymbol{x}-\overline{\boldsymbol{x}})$ (in the symmetrical Stratonovich interpretation). The spatial Fourier transform of Eq. (17),

$$
\partial_{t} C_{\boldsymbol{k}}(t)+2 \operatorname{Re} \eta_{\boldsymbol{k}} C_{\boldsymbol{k}}=F_{\boldsymbol{k}}
$$

has the steady-state solution

$$
C_{\boldsymbol{k}}=F_{\boldsymbol{k}} /\left(2 \operatorname{Re} \eta_{\boldsymbol{k}}\right)
$$

Since both $F$ and $\eta$ depend on all of the Fourier components of $C$, Eq. (26) is a self-consistent integral equation $^{15}$ for $C_{\boldsymbol{k}}$. In practice, the steady-state spectrum is determined as the time-asymptotic state of an initialvalue problem, as reviewed and illustrated in Ref. 16, for 
example. However, the details are not of concern here; I simply assume that the steady-state solution $C_{\boldsymbol{k}}(t=\infty)$ has been determined.

Now consider the effects of a random Gaussian ensemble of zonal flows added to Eq. (13). There is no direct change to the forcing, since zonal flows are not linearly driven. Dissipation of the flows, very important in determining their ultimate saturation level, ${ }^{17,18}$ is ignored since they are here being specified passively at a fixed level. In self-consistent problems, there would be indirect changes in $\eta$ and $\delta f$ because of changes in the spectrum due to mode coupling, but those are ignored in passive problems. One thus analyzes, for fixed background forcing and damping,

$$
\partial_{t} \delta \psi(\boldsymbol{x}, t)+\underline{\delta V_{E, y}(x, t) \partial_{y} \delta \psi}+\eta \star \delta \psi=\delta f(\boldsymbol{x}, t),
$$

where the underlined term was absent in Eq. (13). Let us first assume that the zonal flows are statistically independent of $\delta f$ and repeat the previous calculations with the underlined term included. The left-hand side of Eq. (19) acquires the term

$$
\begin{aligned}
& \partial_{y}\left\langle\delta V_{E, y}(x, t) \delta \psi(\boldsymbol{x}, t) \delta \psi\left(\boldsymbol{x}^{\prime}, t\right)\right\rangle+\left(\boldsymbol{x} \leftrightarrow \boldsymbol{x}^{\prime}\right) \\
& =\partial_{y}\left\langle\left[\delta V_{E, y}(x, t)-\delta V_{E, y}\left(x^{\prime}, t\right)\right] \delta \psi(\boldsymbol{x}, t) \delta \psi\left(\boldsymbol{x}^{\prime}, t\right)\right\rangle
\end{aligned}
$$

the last result following by virtue of translational invariance in $y$. For $x$-independent flows, this term is seen to vanish, so the left-hand side of Eq. (19) is unchanged by such flows. In Eqs. (21) and (22), one must replace $R$ by the random response function $\widetilde{R}$, where $\widetilde{R}$ evolves according to the left-hand side of Eq. (27) and is random because $\delta V_{E, y}$ is. However, because of the assumed statistical independence of $\delta V_{E, y}$ and the background turbulence, the result (23) (with $\widetilde{R}$ on the left-hand side) is again obtained rigorously. One therefore recovers the right-hand side of Eq. (19) unchanged. Thus for $x$ independent flows and vanishing cross-correlations one recovers Eq. (25) and its solution (26), which contain no vestige of the zonal flows. This is true even though the two-time properties of the fluctuations (cf. the response function $R$ ) are modified by those flows. This discussion simply repeats well-known properties of random Galilean invariance. ${ }^{19}$

(I remind the reader that I am postulating symmetry assumptions such that net transport vanishes. In the general case extra terms due to anisotropy arise that are related to net transport; their values are affected by the two-time properties. This emphasizes the point that, in general, transport and fluctuation levels need not be tightly coupled.)

Given the desire to study passive models of the form (12), evidently the only way of finding fluctuation levels dependent on $\delta \widetilde{V}(t)$ is to permit flows statistically correlated to the background turbulence. This is a somewhat unusual and simplistic way of representing the mode-coupling effects introduced by advection with nontrivial space dependence. It might also be viewed as a way of partially capturing the functional dependence between the velocity field and random forcing that would arise in a self-consistent problem. More must be said, however, since in a properly space-dependent model the two-point cross-correlations between zonal flows and background fluctuations vanish as a trivial consequence of homogeneity, since by definition they have different wave numbers. However one looks at it, quantitatively faithful modeling of realistic turbulence requires much more sophisticated models than those discussed here. Nevertheless, it is of some interest to pursue the analysis of a stochastic model with cross-correlations, as it illustrates some general points in statistical turbulence theory and may be useful in future turbulence modeling efforts.

\section{A stochastic-oscillator model}

In order to engage in a preliminary discussion of the qualitative effects of cross-correlations, it is useful to employ as simple a model as possible. Note that if one uses appropriate formulas for $\eta_{\boldsymbol{k}}$ and $F_{\boldsymbol{k}}$, the solution (26) is valid for all wave numbers, from the small, energycontaining ones to the large, dissipative ones. However, that is more information than necessary; because transport is determined mostly by the energy-containing modes, it is adequate to consider a simpler description that merely retains the qualitative behavior of the more general model. For the background turbulence, such a description is

$$
\partial_{t} \delta \psi(\boldsymbol{x}, t)-D \nabla^{2} \delta \psi=\delta f(\boldsymbol{x}, t) .
$$

Here linear effects have been ignored for simplicity, $D$ is the turbulent diffusion coefficient (independent of $\boldsymbol{x}$ ), and $\delta f$ is a random forcing, statistically consistent with $D$, that may be taken to be a centered Gaussian with variance $^{20}$

$$
\left\langle\delta f(\boldsymbol{x}, t) \delta f\left(\boldsymbol{x}^{\prime}, t^{\prime}\right)\right\rangle=F \delta\left(\boldsymbol{x}-\boldsymbol{x}^{\prime}\right) \delta\left(t-t^{\prime}\right),
$$

where $F \doteq 2 \kappa^{2} D$ and $\kappa$ is a constant that measures the strength of the forcing and, thus, the saturation level of the turbulence. For this model, which has $\eta_{\boldsymbol{k}} \rightarrow$ $\nu_{\boldsymbol{k}} \doteq k^{2} D$ (the usual assumption of Dupree's resonancebroadening theory ${ }^{21,22}$ ), the analysis of the fluctuation level goes through as before. One finds a fluctuation level

$$
C_{\boldsymbol{k}}=\kappa^{2} D / \nu_{\boldsymbol{k}}=(\kappa / k)^{2}
$$

this is a familiar mixing-length formula. Of course, in reality wave-number spectra will probably not literally vary as $k^{-2}$ for either large or small $k$; missing from the present model are the details of inertial- and dissipation-range effects, as well as mode couplings between the energy-containing scales. However, those are not important for a qualitative discussion of the effect on 
the macroscopic turbulence level of random zonal flows. The $k$ in formula (31) must be viewed as a typical energycontaining wave number. ${ }^{20}$

I shall now add zonal flows to Eq. (29), but simplify the analog of Eq. (27) further by ignoring the $x$ dependence (hence the shearing effect) of $\delta V_{E, y}: \delta V_{E, y}(x, t)=\delta \widetilde{V}(t)$, $\delta \widetilde{V}$ being a centered Gaussian time series with specified autocorrelation function and rms level $\bar{V}$. Then one may rigorously Fourier analyze Eq. (27) and obtain the forced stochastic oscillator equation

$$
\partial_{t} \delta \psi_{\boldsymbol{k}}+i \delta \omega_{\boldsymbol{k}}(t) \delta \psi_{\boldsymbol{k}}+\nu_{\boldsymbol{k}} \delta \psi_{\boldsymbol{k}}=\delta f_{\boldsymbol{k}}(t),
$$

where $\delta \omega_{\boldsymbol{k}}(t) \doteq k_{y} \delta V(t)$ and

$$
\left\langle\delta f_{\boldsymbol{k}}(t) \delta f_{\boldsymbol{k}}^{*}\left(t^{\prime}\right)\right\rangle=F \delta\left(t-t^{\prime}\right)
$$

In the absence of forcing and damping, Eq. (32) has been studied in depth, both as a model of certain physical processes $^{23,24}$ and, in the limit of time-independent $\delta \omega$, as a nontrivial test case for statistical closures. ${ }^{3,10}$ (For some recent plasma-physics applications of such a model, see Refs. 25 and 26.) If one assumes that the velocity autocorrelation function is characterized by a single autocorrelation time $\tau_{\mathrm{ac}}$, it is well known that the left-hand side of Eq. (32) is parametrized by just two dimensionless parameters, the Kubo number $\mathcal{K}$ and the Reynolds number $\mathcal{R}$. Regimes of the $\mathcal{R}-\mathcal{K}$ space for passive models like Eq. (32) were discussed in Ref. 27. The Kubo number measures the rapidity of the velocity fluctuations. In the present problem, it may be defined as

$$
\mathcal{K} \doteq k_{y} \bar{V} \tau_{\text {ac }}
$$

Although $\mathcal{K}$ is scale-dependent, that dependence is trivial here because $k_{y}$ is a good quantum number; the advection due to the zonal flows does not couple the $k_{y}$ 's. The Reynolds number, a measure of (inverse) dissipation, is defined as

$$
\mathcal{R} \doteq \bar{V} L / D
$$

where $L$ is conventionally taken to be a macroscopic length. However, due to the special status of $k_{y}$ in the present model, it is convenient to take $L=k_{y}^{-1}$. Then with times measured in units of $L / \bar{V}=\left(k_{y} \bar{V}\right)^{-1}$, the Kubo number is just the dimensionless velocity autocorrelation time of the velocity. In dimensionless units, the primitive amplitude equation retains the form (32) with $\delta \omega_{\boldsymbol{k}}$ having unit variance and autocorrelation time $\mathcal{K}$, and with $\nu_{\boldsymbol{k}}=\bar{k}^{2} / \mathcal{R}$, where $\bar{k}^{2} \doteq\left(k_{x} / k_{y}\right)^{2}+1$. The forcing adds a third dimensionless parameter, with strength $F^{\text {(ext) }}=2 \bar{\kappa}^{2} / \mathcal{R}, \bar{\kappa} \doteq \kappa / k_{y}$. Physically $\bar{\kappa}$ would be small, but it may be chosen arbitrarily in the present dynamically linear problem, as it just rescales the steady-state fluctuation level.

Ignoring the $x$ dependence of $\widetilde{V}(t)$ is a very strong assumption; ultimately, of course, it must be included. The motivation here is that interesting statistical effects are already present when decorrelation mechanisms are introduced by any means whatsoever (e.g., random time dependence), so one should understand their consequences first before addressing additional physical and mathematical complications. This philosophy is well established in the theory of stochastic models. ${ }^{23,24,3}$

\section{B. Exact solution of the stochastic-oscillator model}

Equation (32) can be formally solved exactly. I now drop the $\boldsymbol{k}$ labels, which are irrelevant since mode coupling is not treated explicitly. In terms of the random Green's function

$$
\widetilde{G}\left(t ; t^{\prime}\right) \doteq \exp \left(-\int_{t^{\prime}}^{t} d \hat{t}[\nu+i \delta \omega(\hat{t})]\right),
$$

one has

$$
\delta \psi(t)=\int_{-\infty}^{t} d \bar{t} \widetilde{G}(t ; \bar{t}) \delta f(\bar{t})
$$

and

$$
\left\langle|\delta \psi|^{2}(t)\right\rangle=\int_{-\infty}^{t} d \bar{t} \int_{-\infty}^{t} d \bar{t}^{\prime}\left\langle\widetilde{G}(t ; \bar{t}) \widetilde{G}^{*}\left(t ; \bar{t}^{\prime}\right) \delta f(\bar{t}) \delta f^{*}\left(\bar{t}^{\prime}\right)\right\rangle .
$$

In the absence of cross-correlations between $\delta \omega$ and $\delta f$, the factorization

$$
\begin{aligned}
& \left\langle\widetilde{G}(t ; \bar{t}) \widetilde{G}^{*}\left(t ; \bar{t}^{\prime}\right) \delta f(\bar{t}) \delta f^{*}\left(\bar{t}^{\prime}\right)\right\rangle \\
& \quad=\left\langle\widetilde{G}(t ; \bar{t}) \widetilde{G}^{*}\left(t ; \bar{t}^{\prime}\right)\right\rangle\left\langle\delta f(\bar{t}) \delta f^{*}\left(\bar{t}^{\prime}\right)\right\rangle \\
& \quad=e^{-2 \nu(t-\bar{t})} F \delta\left(\bar{t}-\bar{t}^{\prime}\right)
\end{aligned}
$$

is rigorous; then formula (38) evaluates to

$$
\left\langle|\delta \psi|^{2}(t)\right\rangle=\int_{-\infty}^{t} d \bar{t} e^{-2 \nu(t-\bar{t})} F=F / 2 \nu
$$

in agreement with Eq. (26). In the presence of cross-correlations, the Schwartz inequality $|\langle A B\rangle| \leq$ $\left\langle|A|^{2}\right\rangle^{1 / 2}\left\langle|B|^{2}\right\rangle^{1 / 2}$ can be used with $A=\widetilde{G}(t ; \bar{t}) \widetilde{G}^{*}\left(t ; \bar{t}^{\prime}\right)$ and $B=\delta f(\bar{t}) \delta f^{*}\left(\bar{t}^{\prime}\right)$ to conclude that the general result (38) must be less than the correlation-free result (40). In other words, cross-correlations reduce the fluctuation level.

\section{The direct-interaction approximation in the presence of cross-correlations}

For a more quantitative analysis of Eq. (32), one may use a statistical closure approximation. [Although it would be possible in principle to evaluate formula (38) directly, for example by using Monte-Carlo integration, 
the result would provide little insight into the structure of more complicated problems in which closure is essential.] In order to discuss the dependence of the steady-state fluctuation level $I \equiv C(t=\infty)$ on the strength of the cross-correlation $X$ between $\delta f$ and $\delta \omega$, I shall apply the direct-interaction approximation ${ }^{3,10}$ to Eq. (32). That theory is well justified for considering the relative effects of the various time scales inherent in such a statistical problem. (The DIA is not random-Galilean-invariant, ${ }^{19}$ but that is not important here since one is not studying properties of small spatial scales.) Algorithmically, one can obtain the DIA equations by appropriately iterating Eq. (32), treating the stochastically nonlinear ${ }^{3} i \delta \omega$ term as a small correction, then replacing any zeroth-order response functions $R^{(0)}(\tau) \doteq H(\tau) e^{-\nu \tau}$ ( $H$ is the unit step function) that appear on the right-hand side of the evolution equations with the true response function $R(\tau)$ (which must be determined self-consistently). In terms of the functions

$$
\begin{aligned}
C\left(t, t^{\prime}\right) & \doteq\left\langle\delta \psi(t) \delta \psi^{*}\left(t^{\prime}\right)\right\rangle, \\
V\left(t, t^{\prime}\right) & \doteq\left\langle\delta \psi(t) \delta \omega^{*}\left(t^{\prime}\right)\right\rangle, \\
W\left(t, t^{\prime}\right) & \doteq\left\langle\delta \psi(t) \delta f^{*}\left(t^{\prime}\right)\right\rangle, \\
X\left(t, t^{\prime}\right) & \doteq\left\langle\delta f(t) \delta \omega^{*}\left(t^{\prime}\right)\right\rangle, \\
S\left(t, t^{\prime}\right) & \doteq\left\langle\delta \omega(t) \delta \omega^{*}\left(t^{\prime}\right)\right\rangle, \\
F^{(\mathrm{ext})}\left(t, t^{\prime}\right) & \doteq\left\langle\delta f(t) \delta f^{*}\left(t^{\prime}\right)\right\rangle
\end{aligned}
$$

(with $\omega$ being real), the equations of the DIA for nonvanishing cross-correlation $X$ but vanishing mean field are

$$
\begin{aligned}
{\left[R^{(0)}\right]^{-1} R } & +\int_{t^{\prime}}^{t} d \bar{t} \Sigma(t ; \bar{t}) R\left(\bar{t} ; t^{\prime}\right)=\delta\left(t-t^{\prime}\right), \\
{\left[R^{(0)}\right]^{-1} C } & +\int_{0}^{t} d \bar{t} \Sigma(t ; \bar{t}) C\left(\bar{t}, t^{\prime}\right)+\int_{0}^{t} d \bar{t} \underline{\Sigma^{\prime}(t ; \bar{t}) V^{*}\left(t^{\prime}, \bar{t}\right)} \\
& =\int_{0}^{t^{\prime}} F^{(\mathrm{int})}(t, \bar{t}) R^{*}\left(t^{\prime} ; \bar{t}\right)+W^{*}\left(t^{\prime}, t\right), \quad(42 \mathrm{~b}) \\
{\left[R^{(0)}\right]^{-1} \underline{V} } & +\int_{0}^{t} d \bar{t} \Sigma(t ; \bar{t}) \underline{V\left(\bar{t}, t^{\prime}\right)}+\int_{0}^{t} d \bar{t} \underline{\Sigma^{\prime}(t ; \bar{t})} S^{*}\left(t^{\prime}, \bar{t}\right) \\
& =\underline{X\left(t, t^{\prime}\right)}, \\
{\left[R^{(0)}\right]^{-1} W } & +\int_{0}^{t} d \bar{t} \Sigma(t ; \bar{t}) W\left(\bar{t}, t^{\prime}\right)+\int_{0}^{t} d \bar{t} \underline{\Sigma^{\prime}(t ; \bar{t}) X^{*}\left(t^{\prime}, \bar{t}\right)} \\
& =F^{(\mathrm{ext})}\left(t, t^{\prime}\right) .
\end{aligned}
$$

Here

$$
\begin{aligned}
\Sigma(t ; \bar{t}) & \doteq R^{*}(t, \bar{t}) S^{*}(t, \bar{t}), \\
\Sigma^{\prime}(t, \bar{t}) & \doteq R^{*}(t ; \bar{t}) \underline{V(\bar{t}, t),} \\
F^{(\mathrm{int})}(t, \bar{t}) & \doteq C^{*}(t, \bar{t}) S^{*}(t, \bar{t})+\underline{V^{*}(t, \bar{t}) V(\bar{t}, t)} .
\end{aligned}
$$

Terms that vanish with $X$ have been underlined. Note that the left-hand side of the $V$ equation is linear in $V$, so $V \propto X$. The notation $F^{(\mathrm{ext})}$ replaces what was called $F$ in the previous parts of Sec. III in order to avoid confusion with the covariance of the internal noise $F^{(\mathrm{int})}$ that arises from the random advection.

According to Eq. (32), in order to guarantee that $\langle\delta \psi\rangle=0$ one must require

$$
V(t, t)=0 .
$$

[Physically, $V(t, t)$ represents the divergence of the flux due to zonal flows, which would vanish for homogeneous statistics in $y$.] The cross-correlation $X\left(t, t^{\prime}\right)$ may be specified arbitrarily, except that one must respect the Schwartz inequality (a realizability constraint)

$$
\left|X\left(t, t^{\prime}\right)\right| \leq F^{(\mathrm{ext})}(t, t)^{1 / 2} S\left(t^{\prime}, t^{\prime}\right)^{1 / 2} .
$$

Thus, with the strengths of $F^{(\mathrm{ext})}$ and $S$ fixed, the magnitude of $X$ must be less than some maximum value. Another way of seeing this is to note the inequality

$$
\left|V\left(t, t^{\prime}\right)\right| \leq C(t, t)^{1 / 2} S\left(t^{\prime}, t^{\prime}\right)^{1 / 2} .
$$

As we shall see, $C$ decreases as $X$ increases. Since $V \propto X$, one infers a maximum magnitude of $X$ above which Eq. (46) can no longer be satisfied and realizability fails.

Upon recognizing that the first two terms on the lefthand side of Eq. (42d) can be written as $\left(R^{-1} W\right)\left(t, t^{\prime}\right)$, that $W^{*}\left(t^{\prime}, t\right)$ is required on the right-hand side of Eq. (42b), and that $F^{(\mathrm{ext})}\left(t, t^{\prime}\right)=F^{(\mathrm{ext}) *}\left(t^{\prime}, t\right) \equiv$ $F^{(\mathrm{ext}) \dagger}\left(t, t^{\prime}\right)$, one finds

$$
W^{*}\left(t^{\prime}, t\right)=\left[F^{(\mathrm{ext})}+\Delta F\right] \star R^{\dagger},
$$

where

$$
\Delta F \doteq-X \star \Sigma^{\prime \dagger}
$$

and $\star$ denotes convolution. For $X \equiv 0, V$ vanishes as does $\Sigma^{\prime}$, Eq. (47) reduces to $W^{*}\left(t^{\prime}, t\right)=$ $\int_{0}^{t^{\prime}} d \bar{t} F^{(\mathrm{ext})}(t, \bar{t}) R^{*}\left(t^{\prime} ; \bar{t}\right)$, and Eq. (42b) becomes $R^{-1} C=$ $\left[F^{\text {(int) }}+F^{(\text {ext })}\right] \star R^{\dagger}$, or (now dropping the $\star$ )

$$
C=R\left[F^{(\mathrm{int})}+F^{(\mathrm{ext})}\right] R^{\dagger} .
$$

This is the standard form of the two-time spectral balance equation; ${ }^{10,6}$ it shows that $F^{(\mathrm{int})}$ represents the variance of an internal stochastic or "incoherent" noise. For $X \neq 0$, the last term on the left-hand side of Eq. (42b) may be moved to the right-hand side and written as

$$
-\Sigma^{\prime} V^{\dagger} R^{-1 \dagger} R^{\dagger}=-\Sigma^{\prime}\left(R^{-1} V\right)^{\dagger} R^{\dagger} .
$$

From Eq. (42c) one has $R^{-1} V=X-\Sigma^{\prime} S$, or

$$
V=R\left(X-\Sigma^{\prime} S\right) .
$$

Thus Eq. (42b) can be written as

$$
C=R K R^{\dagger},
$$


where

$$
K \doteq F^{(\mathrm{int})}+F^{(\mathrm{ext})}-\underline{\left(X \Sigma^{\prime \dagger}-\Sigma^{\prime} S \Sigma^{\prime \dagger}+\Sigma^{\prime} X^{\dagger}\right)}
$$

is manifestly symmetric. A Schwartz inequality guarantees that the underlined term is positive-definite.

With $C(t) \equiv C(t, t)$, the single-time spectral evolution equation follows from Eq. (42b) as

$$
\begin{aligned}
\partial_{t} C & (t)+2 \nu C \\
& +2 \operatorname{Re} \int_{0}^{t} d \bar{t}\left[\Sigma(t ; \bar{t}) C^{*}(t, \bar{t})+\Sigma^{\prime}(t, \bar{t}) V^{*}(t, \bar{t})\right] \\
& =2 \operatorname{Re} \int_{0}^{t} d \bar{t} F^{(\mathrm{int})}(t, \bar{t}) R^{*}(t ; \bar{t})+2 \operatorname{Re} W(t, t) .
\end{aligned}
$$

Upon noting the formulas (43), one sees that the nonlinear terms [those involving $\Sigma, \Sigma^{\prime}$, and $F^{\text {(int) }}$ ] cancel in Eq. (54). That cancellation arises because the nonlinear part of Eq. (32) conserves $\left\langle\delta \psi^{2}\right\rangle$, and demonstrates the systematic nature of the DIA; such cancellations are usually lost in more heuristic approximations such as resonance-broadening theory. ${ }^{28}$ One thus has

$$
\partial_{t} C(t)+2 \nu C=2 \operatorname{Re} W(t),
$$

with the steady-state solution

$$
I=\operatorname{Re} W / \nu .
$$

For $\Delta F=0$, Eq. (56) reduces to Eq. (40). More generally, the form of Eq. (47), involving the minus sign in Eq. (48), suggests that the presence of cross-correlations reduces the effective forcing and steady-state fluctuation level. One must verify that the sign of the $X \Sigma^{\prime}$ convolution remains positive. As a crude consistency check, note that according to Eq. (42c) $V$ changes sign with $X$, so $X \Sigma^{\prime}$ is invariant under an overall sign change of $X$. ( $R$ is independent of $V$ and $X$.)

It is consistent to impose the symmetries

$$
V\left(t^{\prime}, t\right)=V\left(t, t^{\prime}\right), \quad X\left(t^{\prime}, t\right)=-X\left(t, t^{\prime}\right) .
$$

Then Eqs. (42a) -(42c) can be integrated as a initial-value problem in the domain $t \geq t^{\prime}$ [or $(t, \tau) \geq 0$ ]. [Although Eq. (42d) could also be integrated, it need not be because of the result (47).] I now discuss representative numerical solutions obtained from an extension to passive advection of the code DIA ${ }^{29,30}$ that has been previously used $^{31-33}$ to study various self-consistent statistical problems in plasma physics. To approximate delta-correlated external forcing, $F^{(\mathrm{ext})}(\tau)$ is taken to be proportional to the normalized Gaussian

$$
G(\tau ; \sigma) \doteq\left(2 \pi \sigma^{2}\right)^{-1 / 2} e^{-\tau^{2} / 2 \sigma^{2}},
$$

where $\sigma=(2 / \pi)^{1 / 2} \tau_{\text {ac }}^{(\text {ext })} \ll 1$. As a nontrivial test of the code, one can demonstrate that for $X \equiv 0$ the steadystate fluctuation level is independent of the presence of the nonlinear terms. That can be done by switching those terms on and off in two otherwise identical runs; the result confirms that the code preserves the appropriate symmetries. One can also check the predicted $X=0$ level quantitatively. One may take $\kappa=1\left[F^{(\text {ext })}=2\right]$. Ideally one would recover the result (40), but that is correct only for strictly delta-correlated $F^{(\mathrm{ext})}$. The exact result is

$$
I=\int_{0}^{\infty} d \bar{\tau} F^{(\mathrm{ext})}(\bar{\tau}) R(\bar{\tau})
$$

in practice, one must take account of the finite width of $F^{\text {(ext) }}$, which averages over the initial decaying portion of $R(\bar{\tau})$. For $\nu=\mathcal{O}(1)$ and $\mathcal{K} \ll 1, R$ is expected to decay exponentially (Markovian approximation) with damping rate $\Gamma \doteq \nu+\eta$, where $\eta \approx \mathcal{K}$. The expected level is therefore

$$
I=g\left(\Gamma ; \sigma^{(\mathrm{ext})}\right) F^{(\mathrm{ext})} / \nu,
$$

where

$$
g(\Gamma ; \sigma) \doteq \int_{0}^{\infty} d \bar{\tau} e^{-\Gamma \bar{\tau}} G(\bar{\tau} ; \sigma)=\frac{1}{2} e^{\gamma^{2} / 2} \operatorname{erfc}(\gamma / \sqrt{2}),
$$

$\gamma \doteq \Gamma \sigma . \quad$ For $\nu=1, \mathcal{K}=0.25, \tau_{\mathrm{ac}}^{(\mathrm{ext})}=0.2$, and $F^{\text {(ext) }}=2$, one has $g \approx 0.4294, I \approx 0.859$. As shown in Fig. 1, the code predicts $I=0.876$ for integration with a time step of $\Delta t=0.02$; this agreement is satisfactory. The computed response function is shown in Fig. 2; it compares very well with the Markovian approximation. [In this and the subsequent runs, the arbitrary initial condition $C(0)=1$ was used.]

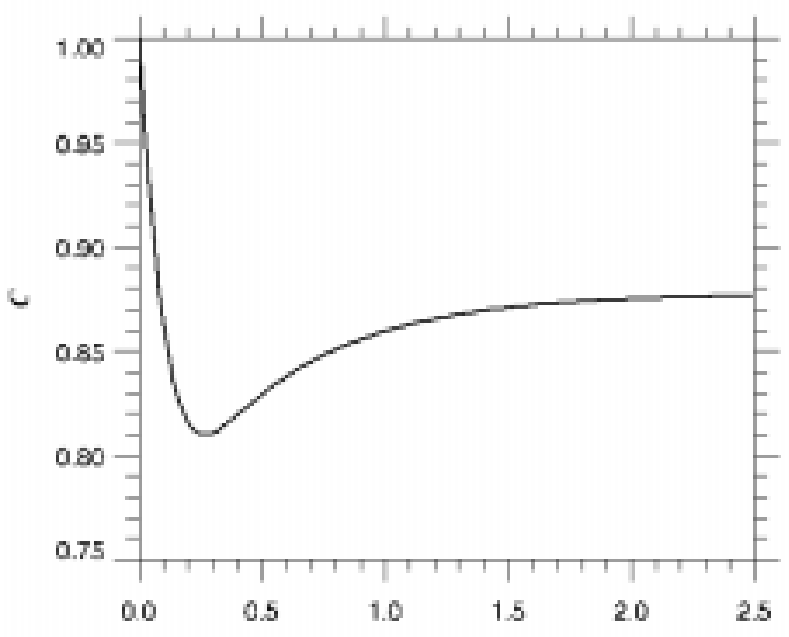

FIG. 1. Time evolution of the fluctuation level $C(t)$ for $X=0, F^{(\text {ext })}=2, \tau_{\text {ac }}^{(\text {ext })}=0.2, \nu=1$, and $\mathcal{K}=0.25$. 


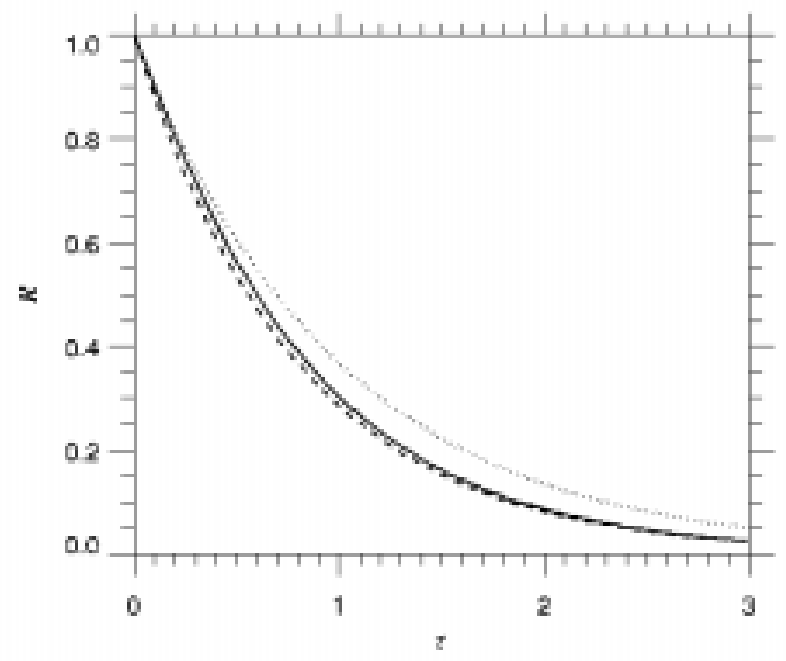

FIG. 2. The computed response function (solid curve) for the parameters $\nu$ and $\mathcal{K}$ of Fig. 1. Dotted curve: $\exp (-\nu \tau)$. Dashed curve: $\exp (-(\nu+\mathcal{K}) \tau)$.

I now discuss a representative solution for $X \neq 0$. Without further research on physics that is beyond the scope of this paper, there is considerable arbitrariness in the forms of $S(\tau)$ and $X(\tau)$. I shall take

$$
\begin{aligned}
S(\tau) & =e^{-|\tau| / \tau_{\mathrm{ac}}}, \\
X(\tau) & =\left[\left(\tau / \tau_{\mathrm{ac}}\right) e^{-|\tau| / \tau_{\mathrm{ac}}} \tau_{\mathrm{ac}}^{-1}\right] \delta,
\end{aligned}
$$

where $\delta$ is an adjustable strength parameter and $\tau_{\text {ac }} \equiv \mathcal{K}$. This choice for the functional form of $X$ ties its time variation to that of $S$, which may not be exactly true in more realistic applications but is satisfactory for purposes of illustration. For the parameters $\tau_{\mathrm{ac}}^{\text {(ext) }}=0.2, F^{(\mathrm{ext})}=2$, $\nu=1, \mathcal{K}=0.5$, and $\delta=2$, Fig. 3 shows $V(\tau)$ (independent of $t)$, Fig. 4 shows the correction $\Delta F$ to $F^{\text {(ext) }}$ [Eq. (48)], and Fig. 5 shows the evolution of $C(t)$ to the forced, dissipative steady state. At short times, $V$ evolves quadratically from $\tau=0$ [recall the initial condition $V(\tau=0)=0$ ] until it integrates over the body of $X$; at long times, the linear and nonlinear dampings force $V$ to decay. $\Sigma^{\prime}(\tau)$, being the product of $R(\tau)$ and $V(\tau)$ [recall $V(-\tau)=V(\tau)]$ possesses a similar shape, being peaked away from $\tau=0$. The convolution $\Delta F \doteq-\Sigma^{\prime} \star X$ averages that peak over a nonzero portion of $X$ selected by $\tau$, giving a nonzero result at $\tau=0$. For large $\tau$, the peak falls on the decaying portion of $X$, so $\Delta F \rightarrow 0$. According to Fig. 4, the overall sign of $\Delta F$ is negative, indicating a reduction in the fluctuation level consistent with Fig. 5.

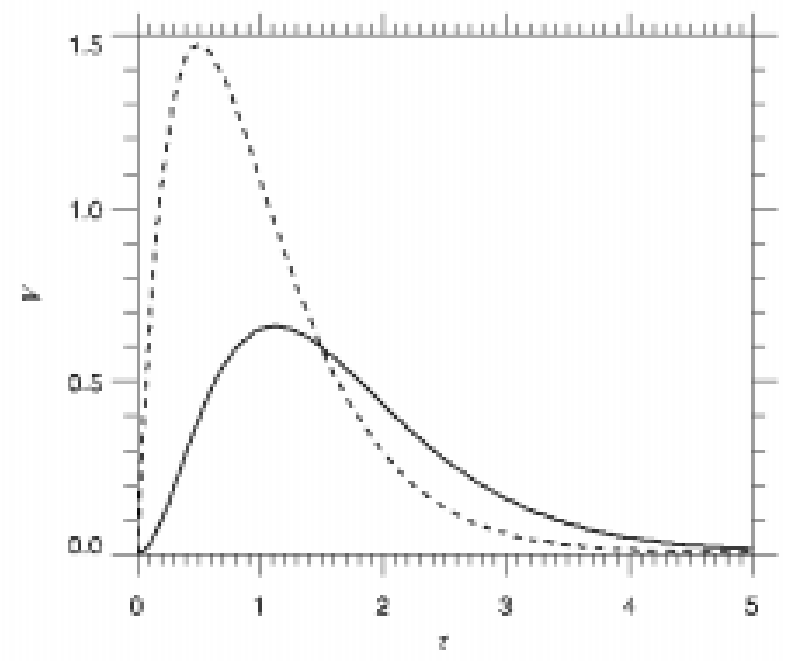

FIG. 3. The calculated $V(\tau)$ (solid curve) for $F^{\text {(ext) }}=2$, $\tau_{\text {ac }}^{(\text {ext })}=0.2, \nu=1, \mathcal{K}=0.5$, and $\delta=2$. Dashed curve: the specified $X(\tau)$.

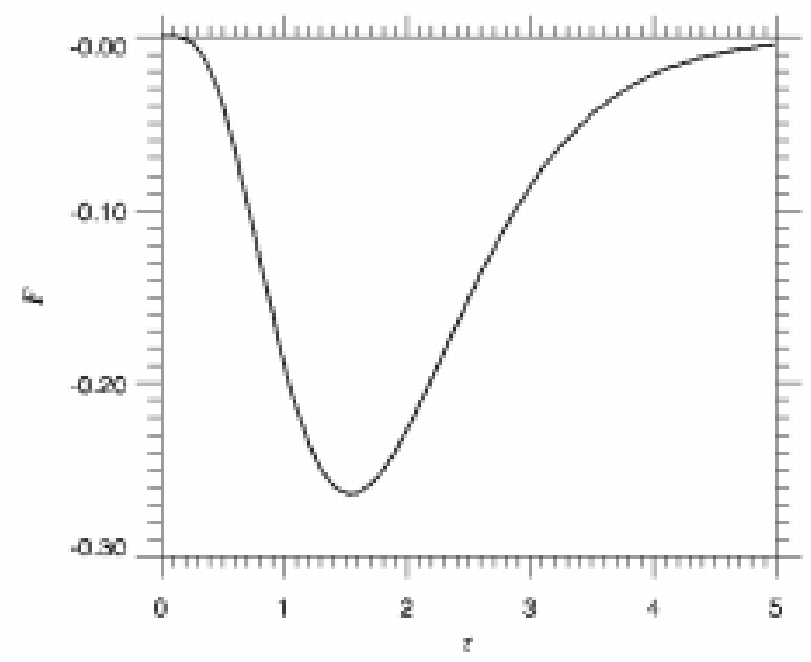

FIG. 4. The nonlinear correction to $F^{(\text {ext })}$ due to nonzero cross-correlation. Parameters are as in Fig. 3. 


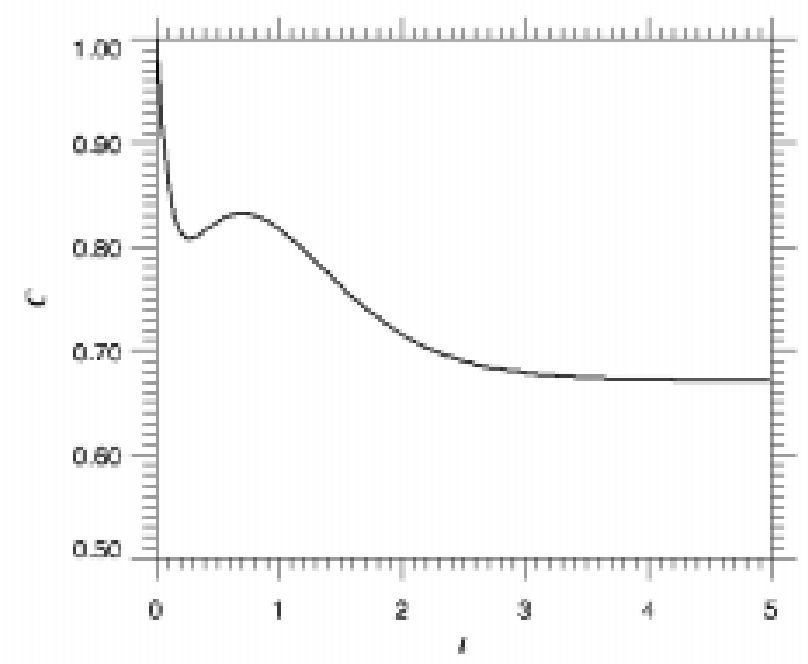

FIG. 5. Evolution of the spectral intensity $C(t)$ to steady state for the parameters of Fig. 3.

The result of performing a series of runs in which the strength $\delta$ of the cross-correlation is varied while all other parameters are held fixed is shown in Fig. 6. For these parameters, realizability evidently fails for $\delta$ slightly bigger than 4 . Figure 6 also shows that the parabola $I=I_{0}\left(1-\alpha \delta^{2}\right)$ gives a good fit. That dependence is easy to understand from the results that $C(t) \propto W(t)$ [Eq. (55)] and that $W$ varies according to Eq. (47); $\Delta F$ [Eq. (48)] is proportional to $-\delta^{2}$ because $\Sigma^{\prime} \propto V$ and $V \propto X$. One is led to the same conclusion by examining the results (52) and (53). It is difficult to perform accurate analytical calculations of the parameters $I_{0}$ and $\alpha$ because the peak in $V$ occurs for $\tau \neq 0$ and is broad, in general. In any event, it does not seem pressing to attempt such a calculation for the present passive model because the real physical interest lies in self-consistent problems in which the shapes of $S(\tau)$ and $X(\tau)$ must be determined as part of the solution.

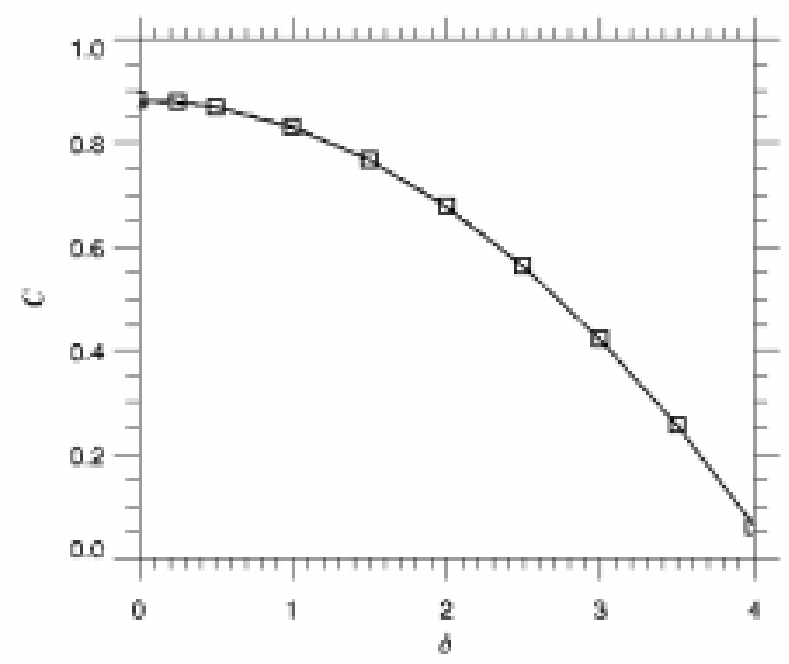

FIG. 6. Dependence of the saturation level $I$ on the strength $\delta$ of the cross-correlations. Other parameters are as in Fig. 3. Solid line: DIA predictions. Dashed line (practically indistinguishable): fit to the parabola $I=I_{0}\left(1-\alpha \delta^{2}\right)$ with $\alpha \approx 0.052$.

\section{DISCUSSION}

In this paper I focused on two facets of fluctuation levels in stochastic models of passive advection (possessing sufficient symmetry so that net transport vanishes): (i) the role of small-scale velocity shear; (ii) the importance of cross-correlations in particular time-dependent models.

First, I recalled in Sec. II the result of Ref. 5 that saturation levels are in general not determined by the rms shear $\omega_{s}$, in contradiction to a widespread current belief. Specifically, with $\omega_{f}$ being the effective frequency of the random velocity, the limit $\omega_{f} \rightarrow \infty$ was treated rigorously by means of the rapid-change model. The effective shearing rate $\omega_{\text {eff }}$ based on a theory of small-scale eddy distortions is proportional to $\omega_{s}$ and vanishes as $\omega_{f} \rightarrow \infty$, both features implying that such a theory cannot determine the saturation level. Instead, that level is determined from the properties of the long-wavelength portion of the spectrum, which lead to the balance $D \sim \gamma / \bar{k}^{2}$, where $\bar{k}^{-1}=L_{\mathrm{c}}$ is the correlation length of the turbulence.

Second, I turned in Sec. III to discussions of Langevin models involving background turbulence plus additional random velocity fields depending only on time. For such models the additional velocity fields do not change the fluctuation level in the absence of cross-correlations between the flows and the background turbulence, regardless of the correlation time of the flows, even though those flows do affect the two-time characteristics of the correlation functions. Although general Langevin models involve wave-number-dependent turbulent damping, a simpler Langevin model comprising a constant diffusion co- 
efficient and statistically consistent Gaussian forcing behaves qualitatively similarly. That approximation leads to a stochastic-oscillator type of model containing only random time dependence; that model was analyzed both exactly and with the direct-interaction approximation. It was shown that in the presence of cross-correlations the fluctuation level is reduced. This result is obtained for a stochastic model that contains no shearing effect (as it involves no $\boldsymbol{x}$ dependence); the reduction depends on the magnitude of the cross-correlations, which in the passive model can be varied independently of the autocorrelation time of the velocity field.

Compelling evidence that zonal flows influence the ultimate level of turbulence ${ }^{34}$ can be found in the computer simulations of Lin. ${ }^{17,18}$ However, the details of the self-consistent statistical interactions that lead to the ultimate steady state may be difficult to untangle; formalisms that focus only on correlation times or lengths or on the dynamics of small-scale eddies are inadequate, as can be seen from the results of Sec. II, and cannot lead to accurate quantitative predictions of fluctuation and transport levels. The details of the $x$ dependence of the flows are clearly important, even if it is not rms shear that controls fluctuation levels. The self-consistent magnitude of the (functional) cross-correlations between zonal flows and background turbulence will depend, in part, on the general structure of the statistical interactions in a way similar to the above model with cross-correlations, and, in part, on the autocorrelation time of the turbulence, which will be affected by the long-wavelength dynamics of the random flows. Sufficient information is contained in the DIA, and a self-consistent DIA analysis of a physical model containing random zonal flows is a natural next step. In such problems, coupling between many Fourier amplitudes presents a substantial additional complication; nontrivial numerical work is required.

It must be emphasized that neither the (rigorous) analyses of the rapid-change model in Ref. 5 and Sec. II, the DIA calculations in Sec. III C, nor more general DIA analyses make any use of the so-called "two-point theory" (a misnomer ${ }^{35}$ ) as it is understood in the context of Dupree-style renormalizations ${ }^{9,36}$ and that has been used, for example, in previous analyses of twodimensional turbulence ${ }^{37}$ and the effects of flow shear on correlation times and lengths. ${ }^{38-41,2}$ That approximation has been analyzed extensively ${ }^{42,35,43,5}$ and shown to be inapplicable to calculations of macroscopic spectral properties such as correlation scales, fluctuation intensity, and transport coefficients. In brief, the issue is that the two-point theory uses approximations appropriate for only the smallest scales of turbulence, and those cannot be extrapolated to the energy-containing scales that dominate transport. For detailed discussion, see Ref. 5, where it was shown how the two-point theory predicts the wrong answer (both qualitatively and quantitatively) for the physically relevant and exactly solvable rapid-change model. This does not mean that useful, experimentally relevant predictions can never emerge from such analy- ses, because important trends are highly constrained by dimensional consistency, but one must be careful to distinguish dimensional results from refined calculations of details. The present work (on very simplified models) demonstrates how one can elucidate some of those details. Unlike the two-point theory, the Markovian closures mentioned in Sec. III A and the direct-interaction approximation used in Sec. III C are very well suited to that end, as they can deal with the entire gamut of scales, including in particular the energy-containing ones.

Finally, although the importance of the DIA for providing conceptual insights has been widely recognized, it is frequently considered to be too complicated for use in practical calculations. In general, realizable Markovian approximations ${ }^{13,31-33}$ provide a viable alternative. However, the passive stochastic-oscillator model studied in Sec. III is a case (nontrivial in the presence of crosscorrelations) in which the DIA can be quantitatively studied without difficulty. That analysis is therefore interesting in its own right.

\section{ACKNOWLEDGMENTS}

I am very grateful to M. Beer, T.-S. Hahm, M. Ottaviani, and $\mathrm{W}$. Lee for questions and comments on the first draft of the manuscript that led to material improvements in the presentation. This research was supported by the U.S. Department of Energy Contract No. DEAC02-76-CHO-3073.

${ }^{1}$ P. W. Terry, Suppression of turbulence and transport by flow shear, Rev. Mod. Phys. (1999) (in press).

2 T. S. Hahm, M. A. Beer, Z. Lin, G. W. Hammett, W. W. Lee, and W. M. Tang, Phys. Plasmas 6, 922 (1999).

${ }^{3}$ R. H. Kraichnan, J. Math. Phys. 2, 124 (1961), erratum: J. Math. Phys. 3, 205 (1962).

${ }^{4}$ J. A. Krommes and M. Ottaviani, Phys. Plasmas 6, 3731 (1999).

5 J. A. Krommes, Phys. Plasmas 4, 655 (1997).

${ }^{6}$ J. A. Krommes, Phys. Rep. 283, 5 (1997).

${ }^{7}$ R. H. Kraichnan, Phys. Rev. Lett. 72, 1016 (1994).

${ }^{8} \mathrm{~A}$ consistent interpretation of formula (3a) requires that $\delta(\tau=0)=\left(2 \tau_{\mathrm{ac}}\right)^{-1}$. One way of thinking about the delta function is as the limit $\delta(\tau)=\lim _{\tau_{\mathrm{ac}} \rightarrow 0}\left(2 \tau_{\mathrm{ac}}\right)^{-1} \times$ $\exp \left(-|\tau| / \tau_{\mathrm{ac}}\right)$. Related discussion of the rapid-change model was given by R. H. Kraichnan, J. Fluid Mech. 75, 657 (1976).

${ }^{9}$ T. H. Dupree, Phys. Fluids 15, 334 (1972).

${ }^{10} \mathrm{~J}$. A. Krommes, in Handbook of Plasma Physics, edited by A. A. Galeev and R. N. Sudan (North-Holland, Amsterdam, 1984), Vol. 2, Chap. 5.5, p. 183.

${ }^{11}$ S. A. Orszag, in Fluid Dynamics, edited by R. Balian and J.-L. Peube (Gordon and Breach, New York, 1977), p. 235. 
${ }^{12}$ C. E. Leith, J. Atm. Sci. 28, 145 (1971).

13 J. Bowman, J. A. Krommes, and M. Ottaviani, Phys. Fluids B 5, 3558 (1993).

${ }^{14}$ R. H. Kraichnan, J. Fluid Mech. 41, 189 (1970).

15 The form of Eq. (25) should not be confused with that of Eq. (5), and the form of Eq. (26) should not be confused with that of Eq. (7b). In Eqs. (25) and (26), $F_{\boldsymbol{k}}=$ $F_{k}^{(\text {int })}+F_{k}^{(\text {ext })}$, where in the rapid-change model [not necessarily assumed in Eqs. (25) and (26)] $F_{k}^{(\mathrm{int})}$ contributes to $D_{-}(\rho)$ via the $\exp (i \boldsymbol{k} \cdot \boldsymbol{\rho})$ term in Eq. (6).

16 J. C. Bowman, Ph.D. thesis, Princeton University, 1992.

17 Z. Lin, T. S. Hahm, W. W. Lee, W. M. Tang, and P. H. Diamond, Phys. Rev. Lett. 83, 3645 (1999).

${ }^{18}$ Z. Lin, Bull. Am. Phys. Soc. 44, 160 (1999).

${ }^{19}$ R. H. Kraichnan, Phys. Fluids 7, 1723 (1964).

${ }^{20}$ More physically, $\delta f$ should be the gradient of white noise, so the forcing is conservative. This detail does not matter in the present rough model, which is intended to describe the saturation of merely a typical energy-containing wave number.

${ }^{21}$ T. H. Dupree, Phys. Fluids 10, 1049 (1967).

22 The resonance-broadening approximation is not always appropriate. For an example in which it fails, see J. A. Krommes, Renormalized dissipation in the nonconservatively forced Burgers equation, submitted to Phys. Plasmas.

${ }^{23} \mathrm{R}$. Kubo, in Lectures in Theoretical Physics, edited by W. E. Brittin and L. G. Dunham (Interscience, New York, 1959), Vol. I, p. 181.

${ }^{24} \mathrm{R}$. Kubo, in Fluctuation, Relaxation, and Resonance in Magnetic Systems, edited by D. ter Harr (Oliver and Boyd, Edinburgh, 1962), p. 23.

${ }^{25}$ J. A. Krommes and G. Hu, Phys. Plasmas 1, 3211 (1994).

${ }^{26}$ J. A. Krommes, Phys. Plasmas 4, 1342 (1997).

27 J. A. Krommes and R. A. Smith, Ann. Phys. (NY) 177, 246 (1987).

${ }^{28}$ T. H. Dupree and D. J. Tetreault, Phys. Fluids 21, 425
(1978).

29 J. A. Krommes, in Statistical Physics and Chaos in Fusion Plasmas, edited by C. W. Horton and L. E. Reichl (Wiley, New York, 1984), p. 241.

30 J. A. Krommes and J. C. Bowman, Bull. Am. Phys. Soc. 33, 2022 (1988).

${ }^{31}$ J. C. Bowman and J. A. Krommes, Phys. Plasmas 4, 3895 (1997).

32 G. Hu, J. A. Krommes, and J. C. Bowman, Phys. Lett. A 202, 117 (1995).

${ }^{33}$ G. Hu, J. A. Krommes, and J. C. Bowman, Phys. Plasmas 4, 2116 (1997).

${ }^{34}$ W. W. Lee (private communication, 1999) has analyzed a few-mode model involving one self-consistently generated zonal-flow mode and shown that the fluctuation level is reduced.

35 J. A. Krommes, Statistical Plasma Physics: Proceedings of the Workshop of the U.S.-Japan Joint Institute for Fusion Theory Program (February 17-21, 1986) (Institute of Plasma Physics, Nagoya U., Japan, 1986), p. 226, see AIP Document no. PAPS PHPAE-04-000-15 for the 15page manuscript "Remarks on the clump theory." Order by PAPS number and journal reference from the American Institute of Physics, Physics Auxiliary Publication Service, 500 Sunnyside Blvd., Woodbury, NY 11797-2999.

36 T. Boutros-Ghali and T. H. Dupree, Phys. Fluids 24, 1839 (1981).

37 T. H. Dupree, Phys. Fluids 17, 100 (1974).

${ }^{38}$ H. Biglari, P. H. Diamond, and P. W. Terry, Phys. Fluids B 2, 1 (1990).

39 T. S. Hahm, Phys. Plasmas 1, 2940 (1994).

40 T. S. Hahm and K. Burrell, Phys. Plasmas 2, 1648 (1995).

41 T. S. Hahm, Phys. Plasmas 4, 4074 (1997).

42 J. A. Krommes, Phys. Fluids 29, 2756 (1986).

43 J. A. Krommes and C.-B. Kim, Phys. Fluids 31, 869 (1988). 